\title{
Labyrinthe
}

$12 \mid 2002$

Numéro 12

\section{Les surréalistes et le musée d'ethnographie}

\section{Vincent Debaene}

\section{OpenEdition}

Journals

Édition électronique

URL : https://journals.openedition.org/labyrinthe/1209

DOI : 10.4000/labyrinthe.1209

ISSN : 1950-6031

Éditeur

Hermann

Édition imprimée

Date de publication : 30 juin 2002

Pagination : 71-94

\section{Référence électronique}

Vincent Debaene, «Les surréalistes et le musée d'ethnographie », Labyrinthe [En ligne], 12 | 2002, mis en ligne le 12 avril 2006, consulté le 15 février 2023. URL : http://journals.openedition.org/labyrinthe/ 1209 ; DOI : https://doi.org/10.4000/labyrinthe.1209

Ce document a été généré automatiquement le 29 septembre 2020

Tous droits réservés 


\title{
Les surréalistes et le musée d'ethnographie
}

\author{
Vincent Debaene
}

1 C'est autour des années 1900 que le "primitivisme » prend naissance en France dans les milieux à la fois culturels, littéraires et artistiques. Les fauves « découvrent » l'art nègre en 1906 (l'événement souvent présenté comme «fondateur» est l'acquisition par Vlaminck d'un masque Fang du Gabon rapidement vendu à Derain), et Picasso date son «illumination» au musée d'Ethnographie du Trocadéro de 1907². Dans le domaine littéraire, on retrouve un intérêt semblable pour l'art "primitif » chez Apollinaire ou Cendrars, qui publie une Anthologie nègre en 1921, compilation de « folklore des peuplades africaines ", avant d'écrire lui-même des "poèmes nègres ». Le milieu des années 1920 marque cependant un tournant par rapport à cette vogue primitiviste, tournant que l'on peut associer à deux phénomènes concomitants : d'une part, l'émergence du surréalisme, d'autre part, la constitution de l'ethnologie comme discipline à part entière (l'ethnologie existe certes déjà, et dispose de son musée depuis 1878, mais elle est, jusqu'aux années 1920 , sous la dépendance des études préhistoriques) $)^{2}$. Cette étude vise à mettre en évidence une proximité historique et thématique entre le surréalisme et l'ethnologie (re)naissante, puis à montrer que cette proximité ne résistera pas aux dynamiques propres de chacun des mouvements. Dans cette perspective, le musée apparaît comme le lieu de cristallisation de cette tension, et on peut lire la brève histoire du front commun des ethnologues et des surréalistes comme celle du passage du musée du Trocadéro au musée de l'Homme ${ }^{3}$.

2 Je rappellerai d'abord brièvement le contexte historique et idéologique général qui a favorisé la rencontre de l'ethnographie et du surréalisme, puis j'aborderai le problème particulier du musée en distinguant le surréalisme orthodoxe de Breton et les surréalistes dissidents de la revue Documents.

Coïncidences et affinités

3 Même si l'on ne considère que la période qui a suivi la guerre, il n'est pas possible de brosser ici un tableau complet du primitivisme ou de l'intérêt général des avant-gardes artistiques pour « la chose nègre » pendant les années 1920, car cet intérêt recouvre les 
manifestations les plus diverses et les plus contradictoires depuis l'exotisme le plus stéréotypé jusqu'à ce qu'Apollinaire appelait la "mélanomanie »" en passant par la découverte du jazz qui connut elle-même plusieurs variantes ${ }^{5}$. Je me contenterai de la coïncidence de quelques dates qui signale la conjonction d'un intérêt renouvelé pour les cultures «primitives» et d'une effervescence culturelle et artistique. En 1923, a lieu la première représentation du ballet la Création du monde ; la musique est de Darius Milhaud, les rythmes sont empruntés au jazz ; le texte de Cendrars est inspiré des mythes Baoulé ; les décors, rideaux et costumes ont été imaginés par Fernand Léger à partir de masques africains (Congo et Côte-d'Ivoire). C'est une des manifestations les plus caractéristiques de la "négrophilie» ambiante et de toutes les ambiguïtés qu'elle peut également comporter (esthétisme, association du jazz et des arts africains dans un même mythe de primitivité régénératrice, etc.). Un an après la publication du premier manifeste, 1925 est une année d'activisme surréaliste (avec, par exemple, le fameux banquet organisé en l'honneur du poète symboliste Saint-Pol Roux); 1925 est aussi l'année où est fondé l'Institut d'ethnologie par Marcel Mauss, Paul Rivet et Lucien Lévy-Bruhl. Par ailleurs, en 1925, le « Tout-Paris » mondain se presse à la Revue nègre au Théâtre des Champs-Élysées pour admirer l'expressivité rythmique et érotique des danses de Joséphine Baker: "Joséphine paraissait en scène, tout juste vêtue de plumes d'autruche sur le postérieur et de quelques bananes suspendues je ne sais où. Elle chantait, mieux vaut dire qu'elle poussait de petits cris; elle dansait, mais cela se bornait à quelques mouvements de l'arrière-train et à un hochement de la tête d'avant en arrière à l'imitation d'une poule. Elle ne faisait rien d'autre. Elle était irrésistible »". Certes, l'image donnée du jazz et de la " culture noire» (c'est avant tout le singulier qui pose problème) est factice, mais cela s'accompagne, chez certains, d'un intérêt réel, ainsi d'André Schaeffner lui-même, futur fondateur de l'ethnomusicologie en France qui participera à la revue Documents, puis à la mission Dakar-Djibouti, et qui publie, dès 1926, le premier livre consacré au jazz'. À cela s'ajoutent de nombreuses rencontres personnelles entre Breton, Eluard, Leiris, Masson, Bataille, Métraux, etc. Dans un entretien de 1961, ce dernier, auteur de l'île de Pâques (1934) et du Vaudou haïtien (1958), revient sur cette période en ces termes " « Ceci se situe vers les années 1924, 1925, 1926, et l'on sait ce que ces années ont représenté dans le mouvement de la pensée. J'y songe encore avec une véritable émotion; c'est une période d'ébullition, de rébellion et nous en étions tous secoués. Pour le dire d'un mot, le surréalisme débutait, et c'est alors qu'il a été le plus vigoureux. Je n'ai pas fait partie du mouvement, mais j'ai connu beaucoup de surréalistes, j'ai eu pour ami Georges Bataille, bref, j'ai suivi ce courant, auquel l'ethnographie a apporté des éléments extrêmement précieux. Brusquement, les peuples exotiques venaient confirmer, en quelque sorte, l'existence d'aspirations qui ne pouvaient pas s'exprimer dans notre propre civilisation ${ }^{8}$

4 L'un des lieux majeurs de cette effervescence est le palais du Trocadéro, qui abrite à la fois le musée des Monuments français et, depuis 1878, le musée d'Ethnographie fondé par E. T. Hamy. C'est un étrange bâtiment sans chauffage ni éclairage : « Logé dans un palais construit pour un tout autre objet, sombre et non chauffé, garni de vitrines improvisées, mal protégées contre la poussière, l'humidité et les insectes, sans salles de manipulation, sans salles de travail, sans laboratoires, sans fichier de collection, le Musée donnait l'impression d'un magasin de bric-à-brac »" Dès 1907, Picasso y avait relevé « une odeur de moisi et d'abandon [qui l'avait] saisi à la gorge » : «J'étais si déprimé que j'aurais voulu partir tout de suite, mais je me suis forcé à rester et à examiner ces masques, tous ces 
objets que des hommes avaient exécutés dans un dessein sacré, magique ${ }^{10}$. Différentes manifestations de tous ordres y sont organisées, en particulier à partir de 1928, date à laquelle Paul Rivet en prend la direction, des expositions bien sûr, mais aussi des galas ou des défilés de mode inspirés de modèles exotiques... Pour trouver des fonds afin de financer la mission Dakar-Djibouti, Rivet organisera aussi un gala de charité au Cirque d'hiver où la légende veut qu'ait eu lieu un combat de boxe « à vide » entre Marcel Mauss et le champion du monde noir américain des poids plumes, Al Brown ${ }^{11}$. Par ailleurs, cette même mission sera en partie financée par Raymond Roussel, auteur " proto-surréaliste " des Impressions d'Afrique.

Outre ce vaste contexte qui mériterait de plus amples analyses, on peut considérer que deux thématiques fondamentales sont communes à l'ethnographie et au surréalisme : le rejet du voyage exotique et «l'objet sauvage ». La nouvelle discipline se constitue en effet autour de deux axes, le terrain et la collecte. Le premier de ces deux impératifs s'oppose d'abord au travail de cabinet, à «l'ethnologie en chambre »; on ne peut plus se contenter de collationner des données récoltées par des missionnaires ou des administrateurs coloniaux, il faut se rendre sur place, rester souvent longtemps, et " pénétrer » la culture observée. Mais le terrain s'oppose aussi au voyage (et, indirectement, au survol impressionniste, au goût superficiel pour la « couleur locale »); le terrain de l'ethnologue commence justement quand le voyage cesse. Quant à l'impératif de collecte, même s'il est une sorte de dégradation empiriste du positivisme dominant, il est d'abord à lire comme un rejet du principe des collections ethnographiques existantes, fondées sur le modèle du « cabinet de curiosités » où seul le degré de bizarrerie commande la sélection de l'objet; il apparait nécessaire au contraire de recueillir un maximum d'objets témoignant des cultures primitives, sans se contenter d'un exotisme tape-à-l'œil. Chez les surréalistes, on trouve un même rejet du voyage romantique et de l'exotisme "à la Loti »; en 1928, Aragon dénonce la rêverie sur les lointains comme une des envahissantes "petites nostalgies bourgeoises » : « L'évasion impossible chacun songe paisiblement à s'évader. [... ] Ils se sont rebâtis un paradis virtuel, qui niche quelque part en Afrique. Anodine transformation des Mésopotamies. Comme n'importe quel fils à papa, l'optimisme est devenu rimbaldien : il ne manquait plus que cela ! $»^{12}$. Dès 1920, on trouve ces mots sous la double plume de Soupault et Breton : « Les affiches enchantées ne nous touchent plus [...]. Les gares merveilleuses ne nous abritent plus jamais ${ }^{13}$. Les conséquences de ce rejet sont, bien sûr, opposées : d'un côté le séjour sur le terrain, de l'autre le choix de Paris, le voyage plus ou moins parodique à Blois et l'étude du « sentiment de la nature aux Buttes Chaumont " (selon le titre de la deuxième partie du Paysan de Paris) ; il reste que ces deux attitudes procèdent bien d'une même source. Par ailleurs, les surréalistes se passionnent pour «l'objet sauvage» (Breton); il s'agit de réhabiliter les arts primitifs dans leur ensemble, et pas seulement africains - Breton comme Eluard leur préféraient d'ailleurs l'art océanien ou mélanésien ${ }^{14}$. En ce sens, le primitivisme surréaliste est un dépassement de la négrophilie ambiante ; il s'agit d'abord de " prendre au sérieux » les productions des arts que l'on dit aujourd'hui premiers, mais aussi de comprendre (pour éventuellement s'en inspirer) les logiques sociales ou psychiques dont elles relèvent. C'est ainsi que les surréalistes précèdent même les ethnographes dans leur dénonciation de la vogue de l'art nègre, ressentie comme superficielle, esthétisante et, qui plus est, passée de mode, en voie de récupération ; à l'enquête lancée par la revue Action en 1920 qui voulait recueillir des « opinions sur l'art nègre », Jean Cocteau répond : «La crise nègre est devenue aussi ennuyeuse que le japonisme mallarméen »; Picasso, quant à lui, se contenta d'un 
cinglant: «L'art nègre? connais pas $»^{15}$. Sans doute ni Cocteau ni Picasso n'étaient surréalistes, mais leurs réponses demeurent significatives de l'état d'esprit d'une certaine frange de l'avant-garde au début des années 1920.

C'est donc en raison de leurs virtualités subversives que les « objets sauvages » suscitent l'intérêt des surréalistes. Comme on le sait, le mouvement est, en grande partie, une réaction à la guerre, qui a montré à quel point la civilisation occidentale pouvait se révéler destructrice, et ces préoccupations rencontrent opportunément le discours de l'ethnologie, car celle-ci insiste justement sur la multiplicité des logiques sociales, sur l'existence d'alternatives à la civilisation européenne (rationaliste, mécanique, aliénante, destructrice), et remet en cause le primat de cette civilisation et de ses productions artistiques. L'ethnologie en voie de constitution participe ainsi au " procès de la pensée » et, en particulier, à la relativisation des valeurs esthétiques occidentales puisqu'elle exige une attitude de "désapprentissage » culturel et une redistribution des catégories comme le beau ou l'art. Par ailleurs, le surréalisme cherche, et trouve parfois, dans les théories ethnologiques de l'époque des arguments pour confirmer ou renforcer ses intuitions fondamentales, en particulier l'idée d'un fond psychique commun à l'humanité. Le premier manifeste prétend à l'universalité : on y lit la volonté de ne pas être une école littéraire de plus mais de toucher au « fonctionnement réel de la pensée » commun à tous les hommes, de tous les lieux et de tous les temps. Jean-Claude Blachère explique ainsi qu'avant de basculer dans la catégorie des auteurs à ne pas lire, le nom de Lévy-Bruhl (pour qui les "primitifs» sont dans un état de communication ininterrompue avec les esprits) est bien une référence ${ }^{16}$, mais rapidement, les surréalistes vont s'éloigner de ces théories qui tracent une frontière entre mentalités primitive et rationnelle ; l'ethnologue qui fournit à Breton ses arguments devient alors Olivier Leroy qui veut rétablir une continuité entre monde " primitif » et « civilisé » et retrouver « la logique expérimentale du savant sauvage ${ }^{17}$. On peut encore noter les allusions de Breton à Freud (qui établit, dans Totem et Tabou, une continuité entre l'artiste et le magicien (chapitre « Animisme, magie et toute-puissance des idées ») ou au célèbre Rameau d'or de Frazer (1908). L'ethnologue britannique y étudie en particulier les fonctions du rêve, et cite plusieurs exemples de cultures " primitives » où le rêve est considéré comme porteur d'une vérité plus élevée que les expériences de la conscience pendant la veille.

André Breton et l'objet ethnographique : la collection contre le musée

7 Une chose réunit avant tout Breton et les ethnographes : l'importance accordée à l'objet matériel. À la différence de l'ethnologie évolutionniste du XIx ${ }^{\mathrm{e}}$ siècle pour laquelle l'objet est avant tout indicateur d'un stade de développement, l'ethnologie des années 1920-1930 envisage l'objet réel comme une sorte de condensation des représentations collectives, de "précipité de la culture », d'« idéogramme matériel $»^{18}$. Comme l'explique Jean Jamin, les origines épistémologiques d'une telle conception sont à chercher à la fois du côté du positivisme sociologique durkheimien, de l'importance accordée par Mauss aux techniques comme conditions de l'état social, et enfin d'une sorte de fonctionnalisme implicite puisque la question posée à l'objet n'est jamais celle du style ou de la variation mais de son intégration, soit à une logique sociale (échange, technique), soit à des représentations collectives qu'on postule toujours unanimes. Quoi qu'il en soit, on retrouve chez les surréalistes un même souci de la matérialité lié au refus très général du dualisme. L'accès à la surréalité exige certes une négation de la réalité mais ce n'est que pour « la soustraire [...] à l'empire de la connaissance rationnelle et de la logique » ${ }^{19} ; c^{\prime}$ est bien la vie réelle et quotidienne dans sa matérialité même qui est à explorer; ce qui est 
chez les ethnologues une forme d'empirisme dégradé est, chez les surréalistes, un principe : l'objet de connaissance et l'objet réel ne font qu'un.

Par ailleurs, Breton retrouve beaucoup de ses conceptions esthétiques dans le discours des ethnographes sur les arts primitifs tel qu'il commence à se dessiner: outre le caractère (supposé) magique de "l'objet sauvage» ou du fétiche, c'est surtout son intégration à la vie quotidienne qui l'impressionne ; qu'un manche de cuiller soit sculpté à l'effigie d'un dieu (car toutes les représentations " anthropomorphes » ne peuvent être d'autre chose qu'un dieu ou qu'un esprit), et c'est une brèche qui s'ouvre entre deux mondes. Autre élément qui séduit les surréalistes: l'activité onirique de l'artiste «primitif» apparaît avant tout impersonnelle; ces sociétés ne connaissent pas le talent individuel (ce qui renforce les convictions de Breton pour qui cette notion est liée à une idéologie romantique et bourgeoise, et qui voit là une réponse à sa recherche du chefd'œuvre sans auteur). Même si de telles idées sont largement remises en cause aujourd'hui, elles étaient à la confluence des deux discours, comme d'ailleurs le sentiment d'urgence face à ces populations en voie d'extinction.

Mais on devine déjà que devant la revendication de scientificité des ethnographes et devant leur souci de fonder la discipline comme entité à la fois épistémologique et institutionnelle, Breton ne pouvait que se raidir, et la question du musée est un excellent révélateur de cette incompatibilité fondamentale. En 1928, Paul Rivet (qui, trois ans auparavant, a fondé l'Institut d'ethnologie avec Mauss et Lévy-Bruhl) est nommé à la chaire d'anthropologie du Muséum, chaire qu'il rebaptise "ethnologie des hommes actuels et des hommes fossiles ", et se voit confier, avec Georges-Henri Rivière, la réorganisation du «bric-à-brac » du vieux musée du Trocadéro. Même si aucun texte de Breton de cette époque ne fait directement allusion à cette réorganisation, on peut voir que son discours sur «l'objet sauvage » reproduit la grande opposition nostalgique et moderne entre la collection et le musée. Cette opposition repose sur trois éléments. D'abord, la sélection des pièces. Pour Breton, la collection n'obéit à aucun principe de sélection prédéterminé; elle est d'abord fondée sur le hasard objectif et la charge émotive. De là son indifférence à la valeur, l'ancienneté ou «l'authenticité » des pièces. Dans un article d'avril 1923 intitulé "Le problème des musées", Valéry présentait l'érudition "en matière d'art" comme "une sorte de défaite»: «Elle substitue ses hypothèses à la sensation, sa mémoire prodigieuse à la présence de la merveille; et elle annexe au musée immense une bibliothèque illimitée. Vénus changée en document. $»^{20}$ Breton radicalise en quelque sorte ce constat en l'étendant au principe même du choix des objets : le savoir livresque ne doit pas y intervenir. Aussi le discours de Breton à l'égard des ethnologues se durcira-t-il avec les années; il dénonce leur "scientisme", leur regard " glacé » et distant, « leurs appareils à tout détecter, sauf précisément ce qui [...] importe, soit ce qui détermine la fusion de l'esprit et du cœur dans un moule verbal ou plastique $»^{21}$. Selon Breton, pour comprendre telle ou telle culture primitive, il faut avoir «l'œil non prévenu », " non instruit de ce qu'il va voir $»^{22}$ : «Les savantes gloses [des] spécialistes des groupes ethniques [...] se dérobent à toute approche sensible de l'objet considéré. Peut-on douter que ce mode d'appréhension qui présuppose le détachement et la froideur, constitue a priori un obstacle insurmontable à la connaissance ? $»^{23}$

10 Second grief de Breton à l'encontre du musée: l'organisation. Qui dit musée dit classement, rationalisation. Or on peut, sans grand risque d'erreur, supposer que ce qui séduisait les surréalistes dans le musée d'Ethnographie du Trocadéro, c'était justement 
l'absence de classement, l'aspect «magasin de bric-à-brac ». À partir de 1928, Paul Rivet et Georges-Henri Rivière distinguent les vitrines «ethnogéographiques » et les vitrines « synthétiques » qui illustrent certains thèmes comme la royauté ou l'initiation : alors que le vieux «Troca » était une brèche dans l'univers confiné des galeries, voilà que, là aussi, on se met à ranger « des unités de plaisir incompatibles sous des numéros matricules, et selon des principes abstraits " ${ }^{24}$. À l'inverse, la collection ne connaît d'autre principe de classement que la prédilection personnelle. Celle de Breton lui-même, qui occupait plusieurs murs de son atelier de la rue Fontaine et qui est aujourd'hui en partie reconstituée et présentée au public au musée national d'Art moderne du Centre Pompidou, faisait voisiner les arts primitifs, les objets trouvés, les objets naturels et les œuvres des contemporains (Braque, Miro, Dali) ${ }^{25}$. Ainsi, pour Breton, le musée ethnographique, sous prétexte d'objectivité, présente les objets dans une équivalence, une indifférence inacceptables. Il s'avère incapable de restituer la puissance poétique de l'objet, alors que l'essentiel, pour l'auteur de Nadja, est avant tout de respecter son rayonnement et sa capacité à métamorphoser, en retour, l'esprit.

11 Enfin, Breton reprend à son compte les récriminations habituelles contre «la cohue des badauds, véritable torrent de lave », « l'interminable défilé des ahuris » et « les cargaisons de touristes congénitalement blasés ${ }^{26}$. Pour Breton comme pour bien d'autres, le dialogue entre l'objet et le poète n'est possible que dans le silence du temple. On retrouve dans cette opposition entre le privé et le public l'une des grandes nostalgies de la modernité : le musée achève de dépayser l'objet et, à l'inverse de la collection qui peut, dans une certaine mesure, corriger cette perte de la sacralité et, par une fréquentation intime, restaurer «l'ambiance cultuelle dont il émane" et rétablir la «chaîne émotionnelle » qui a été brisée ${ }^{27}$, il multiplie les médiations, mais "rien [n'est] moins propice à l'appréhension en profondeur [de l'œuvre du "primitif»] que de devoir en passer par le regard trop souvent glacé de l'ethnographe qui croirait, sinon déchoir, du moins faillir à ses disciplines s'il se portait vers elle avec quelque ardeur ou même s'il se montrait [...] moins rebelle à l'émotion. $»^{28}$

Pour être complet, il faut encore ajouter qu'à cette série d'oppositions entre surréalistes et ethnographes s'ajoute un autre conflit, d'ordre politique cette fois, autour de la question coloniale. Au début des années trente, en effet, l'ethnologie est, de fait, solidaire de l'entreprise coloniale; le musée d'Ethnographie du Trocadéro participe d'ailleurs à l'exposition de 1931. Les surréalistes y sont au contraire violemment opposés (Breton, Eluard, Péret, Dali sont alors membres du PCF); ils diffusent aussi massivement que possible le tract «Ne visitez pas l'Exposition coloniale » et participent activement à la contre-exposition organisée par la Ligue anti-impérialiste intitulée «La vérité sur les colonies » où Paul Eluard et Yves Tanguy font voisiner des "fétiches » africains et des « fétiches » occidentaux, ex-voto, ou statues de Vierge à l'Enfant.

On peut noter, pour conclure, qu'il ne faut sans doute pas rabattre trop rapidement l'opposition entre Breton et les ethnographes sur une banale opposition entre science et poésie, et la réduire à un conflit inévitable entre objectivité et subjectivisme esthète (et ce, malgré l'anecdote des pois sauteurs du Mexique diffusée par Roger Caillois ${ }^{29}$ ) ; comme le signale Jean-Claude Blachère, Breton avait une connaissance relative des textes des ethnologues sur les arts primitifs. On doit plutôt lire dans ce conflit une opposition entre deux modes de connaissance. Pour Breton, renoncer à l'enchantement est une perte non seulement du point de vue de l'émotion, mais aussi du point de vue de la connaissance : 
"Je ne prêche pas ici l'inintelligibilité. Je dis que le besoin de comprendre est limité en nous comme le reste, ne serait-ce que par l'effort auquel il nous astreint ». À propos de Rimbaud, Breton se souvient des derniers mots de la «Lettre-Océan » d'Apollinaire, « Tu ne connaîtras jamais bien les Mayas", qui sonnent comme une admonestation aux ethnologues; il faut maintenir la "part de l'énigme », ce " coin de voile qui demande expressément à ne pas être levé ", faute de perdre dans un écrasement du désir ce qui faisait l'intérêt même de la quête ${ }^{30}$. Le savoir livresque et la collection de bois morts exposée au musée demeurent extérieurs à la culture qu'il s'agit d'investir. Il faut tâcher au contraire de progresser du dehors vers le dedans, de passer de la perception superficielle des aspects géographiques, sociaux et culturels des sociétés primitives à la connaissance intime de l'esprit qui explique, en dernier ressort, l'organisation sociale et la production culturelle. C'est sans doute là tout ce qui oppose une connaissance fondée sur l'observation (même participante) et une connaissance conçue avant tout comme activité de l'esprit désirant.

Les surréalistes dissidents : le « musée pour rire » de la revue Documents (1929-1930)

En 1929, juste avant la publication du Second Manifeste du surréalisme, un mouvement de révolte gagne plusieurs des membres du groupe de Breton. Des exclusions sont prononcées. À la tête de la dissidence, on trouve Georges Bataille qui n'appartient pourtant pas au mouvement, mais pour qui les surréalistes sont «des emmerdeurs idéalistes ». Il est rejoint par Leiris, Desnos, Limbour, Queneau, Vitrac, Prévert, tous signataires du pamphlet "Un cadavre", adressé à Breton sur le modèle du brûlot que celui-ci avait naguère rédigé contre Anatole France ${ }^{31}$. Ces surréalistes dissidents se retrouvent autour d'une éphémère revue, Documents, publiée pendant deux ans et qui compta quinze numéros ${ }^{32}$. Cette revue porte en sous-titre le programme "Doctrines, archéologie, beaux-arts, ethnographie» (qui après quelques numéros deviendra "Archéologie, beaux-arts, ethnographie, variétés »). Elle repose sur la collaboration des surréalistes dissidents et de plusieurs ethnologues ou futurs ethnologues comme Griaule, Schaeffner, ou Rivet et Rivière qui réorganisaient le musée du Trocadéro, et dont le travail allait aboutir à la fondation du musée de l'Homme (décidée en 1934 ; le musée sera inauguré en 1938). Georges Bataille en est le secrétaire général et Michel Leiris, secrétaire de rédaction. On ne peut guère parler de projet commun à propos de cette publication à l'éclectisme revendiqué ; cela dit, les contributions à la revue s'ordonnent presque toutes autour d'une thématique centrale: les questions esthétiques en général, et tout particulièrement la question du musée. On peut considérer que Documents franchit un pas de plus par rapport au surréalisme orthodoxe ; il ne s'agit plus seulement de relativisation des valeurs esthétiques occidentales, mais bien d'une relativisation des valeurs esthétiques en général. Bataille veut " dire oui à tout ", donner à voir, à lire et à penser l'hétéroclite, l'irritant, l'inquiétant. Toutes les formes d'expression, les plus «élevées » comme les plus « populaires »- distinction que, bien entendu, Bataille rejette -, méritent l'attention et doivent être considérées comme des formations symboliques d'un intérêt égal.

La revue adopte donc une esthétique de la juxtaposition, du collage ; elle devient une sorte de «musée pour rire ", pour reprendre l'heureuse expression de James Clifford ${ }^{33}$. S'y trouvent associés des reproductions de toiles de Picasso, des gravures du XviII ${ }^{\mathrm{e}}$ siècle représentant des monstres, des études comparatives des différentes couvertures de Fantômas, des photographies des abattoirs de Paris, d'autres, de gros orteils horriblement agrandis, des masques africains, des peintures médiévales... On retrouve ce parti pris 
iconoclaste dans le "Dictionnaire critique ", élaboré au fil des numéros, rédigé surtout par Bataille, Leiris et Griaule, et qui propose toutes sortes de définitions surprenantes et provocatrices Ainsi à l'entrée «HOMME », on trouve la « définition » suivante (qui est en fait une citation d'un article du Journal des débats consacré aux travaux d'un chimiste anglais, Charles Henry Maye) :

«[...] assez de fer pour fabriquer un clou de grosseur moyenne et du sucre pour sucrer une tasse de café. Le phosphore donnerait 2200 allumettes. Le magnésium fournirait de quoi prendre une photographie. Encore un peu de potasse et de soufre, mais en quantité inutilisable. Ces différentes matières premières, évaluées aux cours actuels, représentent environ une somme de 25 francs $^{34} »$.

16 À l'entrée « MUSÉE », on lit :

«[...] les salles et les objets d'art ne sont qu'un contenant dont le contenu est formé par les visiteurs : c'est le contenu qui distingue un musée d'une collection privée. Un musée est comme le poumon d'une grande ville : la foule afflue chaque dimanche dans le musée comme le sang et elle en ressort purifiée et fraîche. Les tableaux ne sont que des surfaces mortes, et c'est dans la foule que se produisent les jeux, les éclats, et les ruissellements de lumière décrits techniquement par les critiques autorisés ${ }^{35} »$.

17 À Documents, les ethnographes et l'avant-garde se retrouvent sur deux points : un même réalisme agressif et une même volonté de contextualisation. L'idéologie qui préside à la réorganisation du musée du Trocadéro est foncièrement anti-esthétique ; «le Trocadéro ne sera pas plus un musée des beaux-arts que Documents ne sera une Gazette des beaux-arts. Pas un instant Rivière n'envisage de faire concurrence au Louvre $»^{36}$. Le titre de la publication est d'ailleurs, à lui seul, une revendication : un document est un objet dénué de valeur artistique. La déploration de Valéry « Vénus changée en document » aurait pu servir de mot d'ordre aux animateurs de la revue. Pour Rivet et Rivière, il n'est même pas question d'arts primitifs, mais d'ethnographie (en 1928, la nomination de Paul Rivet à la chaire d'anthropologie du Muséum avait d'ailleurs, de fait, placé le musée du Trocadéro sous la tutelle du Muséum d'histoire naturelle). Dès le premier numéro, dans un article qui présente les travaux muséographiques à venir, Georges-Henri Rivière écrit :

« À la suite de nos derniers poètes artistes, artistes et musiciens, la faveur des élites se porte vers l'art des peuples réputés primitifs et sauvages. [...] Ceci provoque dans l'ethnographie d'étranges incursions, accroît une confusion qu'on prétendait réduire. Le Trocadéro rénové pouvait se fonder sur ce contresens, devenir un musée des beaux-arts, où les objets se répartiraient sous l'égide de la seule esthétique. Pauvre principe à la vérité, qui n'aboutit qu'à distraire du tableau et au hasard, quelques-uns seulement de ses éléments essentiels ${ }^{37}$ ».

18 Quelques mois plus tard, Leiris dénonce quant à lui l'esthétisme muséographique qui transforme « un masque ou une statue construite en vue de fins rituelles et compliquées en vulgaire objet d'art » :

«Ce qui est beau dans un tel art, ce n'est pas son côté exotique, ce n'est pas non plus ce qu'il contient de strictement moderne (ce modernisme n'est qu'une pure coïncidence), mais d'abord qu'il ne constitue pas un Art à proprement parler. Il semble, en effet, éminemment absurde d'appliquer à des productions si claires, si spontanées, cet affreux mot à majuscule qu'on ne devrait écrire qu'avec une plume pleine de toiles d'araignées ${ }^{38} »$.

19 Les ethnographes et les surréalistes dissidents ont donc en commun une même volonté de tout dire, de tout montrer: l'ethnologue qui collecte ne doit rien exclure et ne doit surtout pas se conformer à des critères esthétiques; l'ethnographie, écrit Griaule, doit "se méfier du beau, qui est bien souvent une manifestation rare, c'est-à-dire 
monstrueuse, d'une civilisation [...], et elle ne refusera pas une valeur esthétique à un objet parce qu'il est courant et fabriqué en série. ${ }^{39}$ " Conformément aux instructions de Marcel Mauss qui invitait à considérer surtout les manifestations moyennes d'une culture plutôt que ses manifestations monstrueuses ou marginales (grossièrement, la cuiller plutôt que le bijou), la distinction entre le haut et le bas de la culture est abandonnée ; tout, dans une culture, mérite d'être recueilli et de devenir « document ».

Documents est par ailleurs animé par une réaction d'ensemble contre la décontextualisation formaliste. Dans À l'ombre des jeunes filles en fleurs, le narrateur de $L a$ Recherche déplorait la "tyrannie du Particulier » à laquelle l'œuvre doit se soumettre puisque l'église de Balbec ne peut être contemplée que sur la place de Balbec, « inséparable du débouché de la grand-rue, [...] du café et du bureau d'omnibus »; à l'inverse, « la salle de musée symbolise bien mieux par sa nudité et son dépouillement de toutes particularités, les espaces intérieurs où l'artiste s'est abstrait pour créer ${ }^{40}$ ». À l'opposé d'un tel idéalisme, les ethnologues (ou, le plus souvent, futurs ethnologues) insistent sur la nécessité de la réinscription de l'objet dans un lieu, une histoire, une pratique. Griaule raille ainsi " les archéologues et les esthètes [qui admirent] la forme d'une anse, mais [se gardent] bien d'étudier la position de l'homme qui boit. ${ }^{41}$ André Schaeffner est, lui, plus explicite :

"Sur les conservatoires de musique et sur les musées d'art, [le musée d'ethnographie] offre déjà la supériorité de n'avoir rien omis de l'expérience artistique de l'homme. Il admet, de plus, qu'un art serve encore à d'autres fins religion, magie, guerre, chasse, jeu, langage -, qu'il puisse s'isoler mal n'étant point né seul. [...] À côté de l'instrument exposé doit figurer une photographie du joueur de celui-ci ; l'objet muet et sa position entre les mains de qui l'éveille et soudain le multiplie. [...] la reproduction photographique [sera] doublée toujours de son synonyme - le phonographe ${ }^{42}$ ».

21 Encore une fois, il y a là un écho des cours que Mauss professait à l'époque à l'Institut d'ethnologie :

«Un dessin sera joint chaque fois qu'il faudra montrer le maniement de l'objet, un mouvement de la main ou du pied (exemple : pour l'arc et les flèches, il est important de fixer la méthode de lancement par la position des bras, des doigts aux divers moments ; le métier à tisser est incompréhensible sans documents montrant son fonctionnement) ${ }^{43}$ ”.

Les analyses de Walter Benjamin dans l'Euvre d'art à l'époque de sa reproduction mécanisée, quoique postérieures à la revue Documents puisqu'elles datent de 1936, peuvent être lues comme l'expression synthétique de ce refus de la décontextualisation. S'inspirant des analyses de Marx au début du Capital et sur le modèle de l'opposition valeur d'usage/ valeur d'échange, Benjamin reconnaît deux pôles à l'œuvre d'art: sa valeur rituelle inséparable du hic et nunc de l'œuvre, de "son existence unique au lieu où elle se trouve " - et sa valeur d'exposition. Selon lui, l'histoire de l'art peut être lue comme l'histoire de l'opposition entre ces deux pôles. L'objet d'art est à l'origine un objet magique, sacré, intégré dans une tradition et un rituel ; il est porteur d'histoire et est inséparable de son lieu; c'est cette double conjonction, cette « singulière trame de temps et d'espace » qui fait son aura. Mais l'époque moderne est marquée par la « déchéance de l'aura » et par le " poids absolu de la valeur d'exposition ", au détriment de la valeur rituelle qui, pourtant, est première ${ }^{44}$. Autrement dit, et comme le note très justement Denis Hollier, le musée menace l'authenticité de l'œuvre d'art avant même la photographie et la reproduction mécanisée. À la façon dont le marché est un détournement de la marchandise puisqu'il consiste à différer la consommation, à mettre l'objet hors d'usage pour qu'il soit échangé, 
le musée est un détournement de l'œuvre d'art puisque les objets n'y entrent qu'abstraits du contexte de leur valeur d'usage :

«Il y a un contrat symbolique autour de la beauté : de même qu'on ne parle pas d'argent à table, on doit taire au musée les origines laborieuses des objets qu'on expose. Comme l'argent, la beauté n'a pas d'odeur. On passe l'éponge. Ainsi l'exige l'arrivisme esthétique. [...] Les ethnographes de Documents s'en prennent à ce contrat et au refoulement de la valeur d'usage qu'il implique. Ils veulent un musée qui ne réduirait pas automatiquement les objets à leurs propriétés formelles, esthétiques, un espace d'exposition d'où la valeur d'usage ne serait pas exclue, mais dans lequel elle pourrait être non pas simplement présentée, mais exposée, manifestée. Il voudrait déjouer l'alternative qui veut qu'on se serve d'un outil et qu'on regarde un tableau. Que l'entrée de l'outil au musée n'ait pas pour condition qu'il renie ses origines. Au lieu de la remplacer par une valeur d'échange ou d'exposition, cet espace préserverait la valeur d'usage, lui permettrait de survivre à la décontextualisation, coupée de sa fin mais valeur d'usage quand même, une valeur d'usage en sabbatique. À la fois utile et désœuvrée. C'est l'utopie d'un espace où on pourrait, comme disent les Américains, have one's cake and eat it too ${ }^{45}$ "

Cette nostalgie de la valeur rituelle constitue, à Documents, un axe fédérateur; elle est commune aux ethnologues et à l'avant-garde qui partagent l'idée que la chose n'a lieu que sur place et insistent sur la nécessité de la contextualisation.

Cependant, encore une fois, cette rencontre ponctuelle autour de revendications communes ne survivra pas à la dynamique de chacun des mouvements. On peut tenter de retracer l'origine de ces divergences en reprenant les deux points précédents. L'antiesthétisme d'abord. L'anti-esthétisme des ethnologues n'est pas celui de Bataille. Pour les premiers, « tout dire » - ce qui n'est pas tout à fait la même chose que « dire oui à tout » est un impératif scientifique ; les cultures doivent être envisagées comme des ensembles cohérents dont chaque objet témoigne. Pour le second, en revanche, l'anti-esthétisme est avant tout un anti-idéalisme ; le sacrilège est une nécessité pour qui veut rendre compte de la «monstruosité imprésentable du tout». Comme le dit élégamment Denis Hollier, «l'avant-garde ne veut pas du droit de choquer que lui offrent les ethnographes. Si quelqu'un se formalise, on montre son permis. Licence d'ethnographe? Mais que serait un sacrilège dans les limites de la simple raison?» Et plus loin d'ajouter: "Les ethnographes veulent le continu, Bataille veut la rupture $»^{46}$. Les notes de Griaule sur le crachat ou l'intérêt de Schaeffner pour l'instrument de musique «si informe apparaisse-til » ${ }^{47}$ visent en dernière analyse à une réinscription dans le tout d'une culture ; leur antiesthétisme est d'abord statistique; si le beau est écarté, c'est qu'il est monstrueux, exceptionnel, hors de la commune mesure; pour Bataille, au contraire, si le beau est écarté, c'est que, paradoxalement, il est la commune mesure et ce sera la fonction du document que d'exposer le «caractère commun de l'incongruité personnelle et du monstre $^{48}$ ».

Quant à l'exigence de contextualisation, elle masque elle aussi une divergence plus profonde car, à Documents, « la nostalgie de la valeur d'usage suit deux pentes différentes, l'une profane, l'autre sacrée " ${ }^{49}$. Pour les ethnologues, retrouver la valeur d'usage, c'est retrouver la valeur technique, sociale, économique de l'objet, reconstituer le contexte et le circuit où il s'intègre. Pour Bataille, en revanche, retrouver la valeur d'usage, c'est retrouver le sacré. Il y a un maître mot à Documents, celui de fétichisme, et bizarrement, ce ne sont pas les ethnologues qui l'emploient - déjà méfiants sans doute devant ce pseudo-concept un peu trop malléable. Le fétichisme s'oppose à l'esthétisme de l'amateur. 
"Je défie n'importe quel amateur de peinture d'aimer une toile autant qu'un fétichiste aime une chaussure ", écrit Bataille $e^{50}$. Leiris distingue quant à lui le «vrai fétichisme » et le « fétichisme transposé » : le fétiche transposé est précisément celui qui a été mis hors d'usage pour entrer sur le marché, qui a été dégradé en marchandise ou en objet de collection. Giacometti serait un des premiers depuis longtemps à créer de "vrais fétiches " $"$. La réflexion sur le sacré de Leiris et Bataille reprendra quelques années plus tard dans le cadre du Collège de sociologie (1937-1939), mais un sacré sinon détaché de tout support matériel, au moins délivré de la connexion systématique à un objet ; une fois dépassé ce fétichisme du document, version empiriste du «bas matérialisme » de Bataille, le questionnement sur la possibilité d'une science dont l'homme serait à la fois le sujet et l'objet n'en sera que plus vif ${ }^{2}$.

Des préoccupations partagées, donc, mais pas de conciliation possible sur le fond, pas d'«ethnographie surréaliste " ni de "surréalisme ethnographique» en dépit de la séduction exercée par ces deux expressions de James Clifford ; les dynamiques de chacun des «mouvements» - l'ethnographie et l'avant-garde - étaient différentes, voire contradictoires. Griaule, Leiris ou Schaeffner n'étaient pas encore, à l'époque de Documents, les professionnels de l'ethnographie qu'ils deviendront. Comme le note Jean Jamin, « ce que Bataille et ses compagnons retiennent [alors] de l'ethnographie, c'est non pas la méthode ou le savoir, mais une pose: la distanciation, le décentrement, le dépaysement. Ce qu'ils en attendent, c'est, non pas une connaissance positive, mais un choc, un ébranlement, un « dérèglement de tous les sens " ${ }^{53}$. L'ethnographie, de son côté, était en quête de légitimation; elle revendiquait sa scientificité et entamait un processus d'institutionnalisation et de professionnalisation qui devait aboutir en 1937-1938 ${ }^{54}$. Par ailleurs, elle tenait à s'intégrer au projet humaniste et progressiste de la III ${ }^{e}$ République et voulait s'associer à l'entreprise de « civilisation » des colonies, ce qui n'était compatible ni avec l'idéologie politique ni avec l'anticonformisme de l'avant-garde. En 1934, Paul Rivet annonce que le musée du Trocadéro va être rasé et remplacé par une construction grandiose : le palais de Chaillot. L'objectif est clair; il s'agit de dépasser le cosmopolitisme anarchique des années 1920 dans une unité monumentale: «l'humanité»; le changement d'architecture sera à lui seul un signe de cette réorientation. En 1937, le palais est inauguré et l'on peut considérer que lorsque le musée d'Ethnographie devient le musée de l'Homme en 1938, le divorce entre avant-garde et ethnographie est consommé. La revue surréaliste Minotaure (1933-1939) - à laquelle collaborent à la fois Breton et Bataille - apporte peut-être un autre indice de ce divorce : le mot « ethnographie » figure bien au programme, mais à l'exception du deuxième numéro entièrement consacré à la mission Dakar-Djibouti, la "revue à tête de bête " n'accueillera aucune contribution ethnologique dans ses colonnes. Du reste, il est difficile de ne pas voir dans les luxueuses couvertures et les reproductions quadrichromées un retour aux « beaux-arts ».

La proximité du surréalisme et de l'ethnographie naissante est donc indéniable; elle repose sur un certain nombre de soucis partagés et de thématiques communes, ainsi, vraisemblablement, que sur une série de coïncidences historiques; mais les motivations et les dynamiques étaient différentes: le surréalisme attendait des ethnographes des confirmations de ses principes fondamentaux, alors que l'ethnographie, discipline en cours de constitution, se définissait et s'évaluait en se "frottant » aux avant-gardes malgré l'incompatibilité fondamentale des projets. L'histoire de cette proximité est donc en quelque sorte celle du passage du musée d'Ethnographie du Trocadéro au musée de l'Homme; et le bel article de Michel Leiris publié dans La Nouvelle Revue française à 
l'occasion de l'inauguration de 1938 est, à cet égard, révélateur ; il concentre presque tous les arguments des controverses où les ethnologues et l'avant-garde ont été associés, et, malgré sa conclusion résolument progressiste, laisse percer une certaine nostalgie du vieux bâtiment « byzantino-mauresque » que Viollet-le-Duc avait construit :

On peut se demander s'il n'y a pas contradiction dans les termes, tant ce mot « homme» apparaît surprenant accolé au morne terme de «musée », puisque par ce dernier mot est communément désigné le lieu froid où se trouvent déposés des objets que l'homme est fier d'avoir produits, mais qui n'existent plus que pour euxmêmes et séparés de lui. [...] Comment procéder pour que les documents, dont la valeur est liée au fait qu'ils sont choses cueillies sur le vif, puissent garder quelque fraîcheur une fois consignés dans des livres ou mis en cage dans des vitrines? Toute une technique de la présentation devra intervenir comme suite à la technique de collecte, si l'on tient à ce que les documents ne deviennent pas de simples matériaux pour une érudition pesante [...]. Il se peut que d'aucuns regrettent, de l'ancien musée d'Ethnographie, un certain air familier, sans roideur didactique [...]; le nouveau musée de l'Homme n'en marque pas moins un grand pas en avant dans la constitution de ces archives universelles qui ne doivent pas cesser d'être le but de tous ceux qui ont fait de l'étude de l'homme, tant physique que psychique ou social, le point d'application de leur aspiration vers plus de rationnel. ${ }^{55}$

La référence au surréalisme n'a pourtant pas fini de hanter l'ethnologie ; elle resurgira en particulier chez Lévi-Strauss qui fuira la France occupée sur le même navire que Breton et qui, à plusieurs reprises dans la suite, comparera ses méthodes d'élaboration des Mythologiques à la technique du collage, empruntée à Max Ernst ${ }^{56} \ldots$

\section{BIBLIOGRAPHIE}

***, Documents, réimpression, éditions Jean-Michel Place, 1991.

BENJAMIN Walter, «L'œuvre d'art à l'époque de sa reproduction mécanisée » (1936), Écrits français, Gallimard, 1970, pp. 141-171.

BLACHÈRE Jean-Claude, Les Totems d'André Breton. Surréalisme et primitivisme littéraire, L'Harmattan, 1996.

BRETON André, « Flagrant Délit », La Clé des champs, Éditions du Sagittaire, 1953, pp. 134-143.

- «Phénix du masque » (1960), Perspective cavalière, pp. 182-186.

- « Main première » (1962), Perspective cavalière, Gallimard, 1970, pp. 221-225.

CAILLOIS Roger, « Divergences et complicités », La Nouvelle Revue française, n 172, 1967, pp. 686-698.

CLIFFORD James, « Ethnographie, polyphonie, collage », Revue de musicologie, t. 68, n 1-2, 1982, pp. 42-56.

- Malaise dans la culture. L'ethnographie, la littérature et l'art au XXe siècle (1988), École nationale supérieure des beaux-arts, 1996. 
HOLLIER Denis, «La valeur d'usage de l'impossible », introduction à la réimpression de la revue Documents, éditions Jean-Michel Place, 1991, pp. VII-XXXIV.

JAMIN Jean, « Objets trouvés des paradis perdus. À propos de la mission Dakar-Djibouti », Collections passion, éditions Jacques Hainard et Roland Kaehr, musée d'Ethnographie, Neuchâtel, 1982, pp. 69-100.

- « Les objets ethnographiques sont-ils des choses perdues? ", Temps perdu, temps retrouvé, éditions Jacques Hainard et Roland Kaehr, musée d'Ethnographie, Neuchâtel, 1985, pp. 51-74.

- « L'ethnographie mode d'inemploi. De quelques rapports de l'ethnologie avec le malaise dans la civilisation ", Le Mal et la Douleur, éd. Jacques Hainard et Roland Kaehr, musée d'Ethnographie, Neuchâtel, 1986, pp. 45-79.

- « Documents revue. La part maudite de l'ethnographie », L’Homme n¹51, juillet-septembre 1999, pp. 257-266.

LEIRIS Michel, « Du musée d'Ethnographie au musée de l'Homme », la Nouvelle Revue française, 1938, pp. 344-345.

- « De Bataille l'impossible à l'impossible Documents » (1963) ; Brisées, Mercure de France, 1966, pp. 256-266.

- « Le musée de l'Homme, où l'art et l'anthropologie se rencontrent », Réalités, n 182, 1966, pp. 57-63.

LÉVI-STRAUSS Claude, De près et de loin, entretiens avec Didier Éribon, éditions Odile Jacob, 1990.

- « Une peinture méditative », Le Regard éloigné, Plon, 1983, pp. 327-331.

- « New York post- et préfiguratif », Le Regard éloigné, Plon, 1983, pp. 345-356.

MÉTRAUX Alfred, « Rencontre avec les ethnologues », Critique, nº 195-196, 1963, pp. 677-684.

-« Entretiens avec Alfred Métraux », L’Homme, mai-août 1964, pp. 20-32.

RUBIN William (ed.), «Primitivism » in XXe Century Art. Affinity of the Tribal and the Modern, The Museum of Modern Arts, New York, 1984.

VALÉRY Paul, « Le problème des musées », Euvres, vol. 2, Pléiade, Gallimard, 1960, pp. 1290-1293.

\section{NOTES}

1. Des objets qu'il observa lors de sa première visite au musée du Trocadéro, Picasso dira qu'il lui firent comprendre " le sens même de la peinture » : « Ce n'est pas un processus esthétique ; c'est une forme de magie qui s'interpose entre l'univers hostile et nous, une façon de saisir le pouvoir, en imposant une forme à nos terreurs comme à nos désirs. Le jour où je compris cela, je sus que j'avais trouvé mon chemin. » (cité par Françoise Gilot et Carlton Lake dans Vivre avec Picasso, Calmann-Lévy, 1965, p. 249). Sur le primitivisme et

l'art moderne au xx ${ }^{\mathrm{e}}$ siècle, voir l'ouvrage dirigé par William Rubin, «Primitivism » in $\mathrm{xx}^{\text {th }}$ Century Art. Sur le fameux masque Fang acheté par Vlaminck, voir p. 13 ; sur «l'illumination » du Trocadéro, voir l'étude très documentée de Rubin lui-même, en particulier les pages 254 et suivantes.

2. Sur cette « préhistoire » de l'ethnologie française, voir l'ouvrage de Nélia Dias, Le Musée d'Ethnographie du Trocadéro : 1878-1908 : anthropologie et muséologie en France (Éditions du 
CNRS, 1991), ainsi que son édition critique des ouvrages de Ernest-Théodore Hamy, Les Origines du musée d'Ethnographie du Trocadéro ( Les cahiers de Gradhiva », éditions JeanMichel Place, 1988).

3. Ce travail a été, dans une première version, l'objet d'une intervention dans le séminaire de M. Antoine Compagnon sur " Littérature et musée », en mai 2000. Il s'agit surtout d'une tentative de synthèse des travaux de James Clifford, Jean Jamin, Denis Hollier et Jean-Claude Blachère autour de la question particulière du musée.

4. « Mélanophilie ou mélanomanie » (1917), CEuvres en prose complètes, vol. III, Pléiade, Gallimard, 1993, pp. 252-254.

5. Sur cette question, voir dans le numéro récent de L'Homme, « Jazz et anthropologie », $\mathrm{n}^{\circ}$ 158-159, avril-septembre 2001, les contributions de Jean Jamin, Olivier Roueff et Denis Constant-Martin.

6. André Schaeffner, cité par Jean Jamin dans « Objets trouvés des paradis perdus. À propos de la mission Dakar-Djibouti », Collections passion, éditions Jacques Hainard et Roland Kaehr, musée d'Ethnographie, Neuchâtel, 1982, pp. 69-100, p. 76.

7. André Schaeffner, Le Jazz (1926), « Les cahiers de Gradhiva », éditions Jean-Michel Place, 1988.

8. « Entretiens avec Alfred Métraux », L’Homme, mai-août 1964, p. 21.

9. Paul Rivet et Georges-Henri Rivière, chargés de la réorganisation du musée, cités par Jean Jamin dans « Objets trouvés des paradis perdus... », p. 72.

10. Vivre avec Picasso, op. cit., pp. 248-249.

11. Légende rapportée par James Clifford d'après des témoignages de Georges-Henri Rivière (Malaise dans la culture. L'ethnographie, la littérature et l'art au XX $X^{e}$ siècle [1988], École nationale supérieure des beaux-arts, 1996, p. 139).

12. Traité du style (1928), Gallimard, «L'Imaginaire », 1980, pp. 84-85.

13. Les Champs magnétiques (1920), Gallimard, « Poésie », 1971, p. 27.

14. Voir l'explication de William Rubin à ce sujet, p. 55 sqq.

15. Action, $n^{\circ} 3$, réimpression Jean-Michel Place, 1999, p. 24. Il s'agit là des tendances dominantes du discours surréaliste ; dans les faits, l'attitude des surréalistes à la fois face à l'exotisme et face à l'art nègre est parfois plus ambiguë. Voir en particulier le chapitre IV de l'ouvrage de Jean-Claude Blachère, les Totems d'André Breton. Surréalisme et primitivisme littéraire, L'Harmattan, 1996.

16. C'est en 1931 que, au dos d'un catalogue de livres et publications surréalistes édité par José Corti, le groupe établit une liste : «Lisez.../Ne lisez pas... » (Tracts surréalistes et déclarations collectives, 1922-1939, Le Terrain vague, 1980, p. 202).

17. Olivier Leroy, La Raison primitive, essai de réfutation de la théorie du prélogisme, $\mathrm{P}$. Geuthner, 1927.

18. Ces deux expressions sont de Jean Jamin ; la première provient de l'article « Objets trouvés... » (p. 94), la seconde, de l'article « Les objets ethnographiques sont-ils des choses perdues? » (Temps perdu, temps retrouvé, éditions Jacques Hainard et Roland Kaehr, musée d'Ethnographie, Neuchâtel, 1985, p. 68).

19. Ferdinand Alquié, Philosophie du surréalisme, Paris, Flammarion, 1955, p. 98.

20. « Le problème des musées », CEuvres, vol. II, Pléiade, Paris, Gallimard, 1960, pp. 1290-1293.

21. «Flagrant Délit », La Clé des champs, Paris, Éditions du Sagittaire, 1953, p. 140.

22. « Main première » (1962), Perspective cavalière, Paris, Gallimard, p. 221.

23. «Phénix du masque » (1960), Perspective cavalière, pp. 183-184.

24. Valéry, art. cit., p. 1292. 
25. Jusqu'au 24 juin 2002, le mur de l'atelier (celui face auquel Breton travaillait) est présenté dans le cadre de l'exposition «La révolution surréaliste ». On ne peut que noter le caractère paradoxal de cette reconstitution, non seulement parce qu'elle soumet la collection à « l'interminable défilé des ahuris » devant la vitrine, mais surtout parce qu'elle fige le « mur d'objets » tel qu'il était en 1966 à la mort de Breton, alors que, de son vivant, celui-ci n'avait de cesse d'en modifier l'agencement pour découvrir de nouvelles associations et créer de nouvelles rencontres productives - mais une exposition sur le surréalisme doit-elle être une exposition surréaliste?

26. « Flagrant Délit », pp. 137 et 140.

27. « Phénix du masque », p. 184.

28. «Main première », p. 222. On doit cependant noter un malaise persistant des surréalistes face au principe de la collection : celle-ci renvoie non seulement au besoin bourgeois de posséder, mais participe en plus à un pillage qui hâte l'extinction des cultures primitives ( $c f$. Blachère pp. 136-142).

29. Roger Caillois raconte que, peu avant son exclusion du mouvement en 1934, il était entré en conflit avec Breton à propos de l'étrange phénomène de ces pois venus du Mexique et agités d'un mouvement incompréhensible. Caillois, futur ethnologue, aurait proposé d'ouvrir les graines pour vérifier la présence éventuelle d'insectes sous la cosse ce qui était effectivement le cas -, et Breton serait entré dans une violente colère devant un tel rationalisme. Celui-ci s'expliquera plus tard sur cet épisode dans un entretien, précisant qu'il s'agissait d'abord d'« épuiser toutes les conjectures ».

30. « Flagrant Délit », p. 138.

31. Voir Tracts surréalistes et déclarations collectives, 1922-1939, op. cit., pp. 19-25 pour le pamphlet original ; pp. 132-148 pour l'agressif pastiche rédigé par Limbour, Desnos, Bataille, etc.

32. La bibliographie concernant Documents est énorme et, force est de le constater, quelque peu disproportionnée en regard du faible nombre de livraisons qu'a connues la revue ; outre l'excellente introduction de Denis Hollier à la réimpression chez Jean-Michel Place (dont je reprends très largement les analyses), on peut noter l'ouvrage de Georges Didi-Huberman, La Ressemblance informe ou le Gai-Savoir visuel selon Georges Bataille (Macula, 1995), celui de James Clifford, Malaise dans la culture (chapitre 4) et les articles de Michel Leiris (« De Bataille l'impossible à l'impossible Documents ») et Jean Jamin (« Documents revue. La part maudite de l'ethnographie ») (références exactes en bibliographie). 33. « La culture devient chose à recueillir et collectionner et Documents est lui-même une sorte de vitrine ethnographique où sont exposés objets, images, textes, étiquettes, un musée pour rire dont les spécimens sont reclassés en même temps que collectés. » («Ethnographie polyphonie collage », Revue de musicologie, t. 68, n 1-2, 1982, p. 51.)

34. Documents, 1929, n 4, p. 215.

35. Documents, $1930, \mathrm{n}^{\circ}$ 5, p. 300.

36. Denis Hollier, « La valeur d'usage de l'impossible », introduction à la réimpression de la revue Documents, éditions Jean-Michel Place, 1991, p. XV. Comme on le voit, c'est à cette époque que se définissent - pour ne plus vraiment changer - les termes du débat entre ethnologues et « esthètes » tel qu'il se poursuit aujourd'hui autour du transfert des collections du musée de l'Homme et du projet de musée des Arts premiers du quai Branly. Seul Michel Leiris tentera de jeter « les bases d'une ethno-esthétique » dans Afrique noire : la création plastique (1967), mais, comme l'écrit Jean Jamin, « l'approche et les perspectives fortement novatrices » de l'ouvrage sont demeurées en grande partie « lettre morte » alors que, depuis les années 1980, s’est développé un « anti-ethnologisme », un 
« intuitionnisme esthétique » dans l'appréhension et la présentation des œuvres d'art africain, « dont le classement sous la rubrique prétendument nouvelle, mais certainement ambiguë, d'« art premier » ne fait qu'ajouter à la confusion et à l'indétermination auxquelles elles se voient dès lors vouées. » (« Présentation de Afrique noire : la création plastique ", Miroir de l'Afrique, 1996, « Quarto », Paris, Gallimard, pp. 1112-1114.)

37. «Le musée d'Ethnographie du Trocadéro », Documents, 1929, n 1, p. 58.

38. «Civilisation », Documents, 1929, $\mathrm{n}^{\circ}$ 4, pp. 221-222.

39. « Un coup de fusil », Documents, $n^{\circ} 1$, p. 46.

40. Marcel Proust, À l'ombre des jeunes filles en fleurs, Paris, Folio, pp. 227-230 et 213-214.

41. «Poterie », Documents, $1930, n^{\circ} 4$.

42. « Des instruments de musique dans un musée d'ethnographie », Documents, 1929, n 5, pp. 249 et 252.

43. Manuel d'ethnographie (1947), Payot, 1967, p. 17. Ce manuel est la transcription des cours donnés par Mauss à l'Institut d'ethnologie entre 1925 et 1940 sous le titre «Instructions d'ethnographie descriptive à l'usage des voyageurs, administrateurs et missionnaires ».

44. «L'œuvre d'art à l'époque de sa reproduction mécanisée », Écrits français, Paris, Gallimard, 1970, pp. 141 et 147.

45. Denis Hollier, « La valeur d'usage de l'impossible », p. XI.

46. Ibid., p. XIX.

47. "Des instruments de musique dans un musée d'ethnographie », art. cit., p. 248.

48. Georges Bataille, «Les écarts de la nature », Documents, 1930, n 2, p. 82.

49. «La valeur d'usage de l'impossible», p. XIV.

50. Documents, $1930, \mathrm{n}^{\circ}$ 7, p. 489.

51. «Alberto Giacometti », Documents, 1929, $\mathrm{n}^{\circ} 4$.

52. Sur le Collège de sociologie, voir le recueil édité par Denis Hollier en 1979, Le Collège de sociologie, 1937-1939 (réédité en « Folio/essais » en 1995) ainsi que l'article de Jean Jamin, "Un sacré collège ou les apprentis sorciers de la sociologie », Cahiers internationaux de sociologie, $\mathrm{n}^{\circ} 68,1980$, pp. 5-30.

53. «Documents revue. La part maudite de l'ethnographie », L'Homme, $n^{\circ} 151$, juilletseptembre 1999, p. 266.

54. Sur ces questions, voir l'article de Victor Karady, « Le problème de la légitimité dans l'organisation de l'ethnologie historique française ", Revue française de sociologie, vol. XXIII, 1982, pp. 17-35.

55. « Du musée d'Ethnographie au musée de l'Homme », La Nouvelle Revue française, août 1938, pp. 344-345.

56. Voir Tristes Tropiques, chap. 2 et, pour la référence au collage, voir dans Le Regard éloigné, l'article « Une peinture méditative », pp. 327-331.

\section{RÉSUMÉS}

L'Institut d'ethnologie est fondé en 1925 ; en 1928, Paul Rivet et Georges-Henri Rivière sont chargés de réorganiser le vieux musée d'Ethnographie du Trocadéro où les fauves et les cubistes 
avaient « découvert » l'art nègre au début du siècle. En 1934, on décide finalement de raser le bâtiment ; à sa place est érigé le palais de Chaillot où sera inauguré le musée de l'Homme en 1938. Ces quelques années sont marquées par une effervescence culturelle et artistique où se croisent et se mêlent les préoccupations des ethnologues et de l'avant-garde surréaliste. Mais cette proximité n'aura qu'un temps, les exigences de l'avant-garde n'étant guère compatibles avec l'institutionnalisation de la discipline autour du musée de l'Homme. C'est aussi pendant cette période que se mettent en place les oppositions entre ethnologues et « esthètes » telles que l'on peut les observer aujourd'hui encore dans les controverses qui entourent le transfert des collections du musée de l'Homme et le projet de musée des Arts premiers du quai Branly.

Surrealists and the Ethnography Museum

The Institute of Ethnology was founded in 1925 ; in 1928, Paul Rivet and Georges-Henri Rivière were put in charge of reorganizing the old Museum of Ethnography in the Trocadéro where the Fauves and the Cubists had discovered the Negro art at the begining of the century. In 1934, the building was finally destroyed and replaced by the Palais de Chaillot where the Musée de l'Homme would be inaugurated in 1938. These years are marked by an artistic and cultural dynamism where ethnologist's and avant-garde surrealists'interests intersected. But this proximity would not last because the avant-garde's demands were incompatible with the institutionalization of the discipline of ethnology around the Musée de l'Homme. During this period oppositions between ethnologists and "esthetes" also appeared as we may still see it today in the controversies concerning the transfer of the Musée de l'Homme's collections and the Musée des Arts Premiers's project at the quai Branly.

\section{AUTEUR}

\section{VINCENT DEBAENE}

Vincent Debaene est ancien élève de l'École normale supérieure et agrégé de lettres modernes. Ses recherches portent sur les rapports entre anthropologie et littérature. Il enseigne à l'université Paris IV et prépare une thèse sur le récit de voyage ethnographique entre 1930 et 1960. 